\title{
Delay in Treatment of Neonatal Seizures: A Retrospective Cohort Study
}

\author{
Wievineke M.J. Apers ${ }^{a}$ Linda S. de Vries ${ }^{a, b}$ Floris Groenendaal ${ }^{a, b}$ \\ Mona C. Toet ${ }^{a}$ Lauren C. Weeke ${ }^{a}$ \\ aDepartment of Neonatology, Wilhelmina Children's Hospital, University Medical Centre Utrecht, \\ Utrecht, The Netherlands; ${ }^{b}$ Brain Centre, University Medical Centre Utrecht and Utrecht University, \\ Utrecht, The Netherlands
}

\section{Keywords}

Newborn · Anti-epileptic drugs · Seizures ·

Amplitude-integrated electroencephalography

\begin{abstract}
Introduction: Neonatal seizures are common and caused by a variety of underlying disorders. There is increasing evidence that neonatal seizures result in further brain damage. Objective: To describe the time interval between diagnosis of amplitude-integrated electroencephalography (aEEG)confirmed seizures and administration of anti-epileptic drugs (AEDs). Methods: Single-centre retrospective cohort study, with full-term infants ( $n=106)$ admitted to a level III neonatal intensive care unit between 2012 and 2017 with seizures confirmed on 2-channel aEEG and corresponding raw electroencephalography traces, treated with AEDs. The time interval between the first seizure on the aEEG registration and AED administration was calculated. Factors associated with early treatment were analysed. Results: The median time interval of initiating treatment of aEEG-confirmed seizures was 01:50 $\mathrm{h}$ (interquartile range 00:43-4:30 $\mathrm{h}$ ). Treatment of aEEG-confirmed seizures was initiated $<1 \mathrm{~h}$ in
\end{abstract}

(C) 2020 The Author(s)

Published by S. Karger AG, Basel

This article is licensed under the Creative Commons AttributionNonCommercial-NoDerivatives 4.0 International License (CC BY NC-ND) (http://www.karger.com/Services/OpenAccessLicense). Usage and distribution for commercial purposes as well as any distribution of modified material requires written permission.
$34 / 106$ infants (32.1\%), between 1 and $2 \mathrm{~h}$ in 21/106 infants (19.8\%), 2-4 h in 23/106 infants (21.7\%), 4-8 h in 14/106 infants (13.2\%), and $\geq 8 \mathrm{~h}$ in $14 / 106$ infants (13.2\%). Seizures treated $<1 \mathrm{~h}$ were significantly more often recognized by the seizure detection algorithm (SDA) compared to seizures treated $>1 \mathrm{~h}(67 \mathrm{vs} .42 \%, p=0.02)$ and showed more clinical signs ( 79.4 vs. $37.5 \%, p<0.01)$. There was no difference for out-of-office hours ( 23.5 vs. $22.2 \%, p=0.88$ ). Conclusion: With only $32.1 \%$ of the seizures being treated $<1 \mathrm{~h}$, there is room for improvement. Timely treatment occurred more often when seizures were clinical or recognised by the SDA. aEEG is a helpful tool for diagnosing seizures $24 / 7$.

$$
\begin{aligned}
& \text { (c) } 2020 \text { The Author(s) } \\
& \text { Published by S. Karger AG, Basel }
\end{aligned}
$$

\section{Introduction}

Neonatal seizures are a frequent problem in a neonatal care setting and are associated with increased long-term morbidity and mortality. They have a variety of underlying causes and are the most common sign of brain injury [1-3]. Diagnosing seizures is often difficult, as they can be very subtle, indistinctive, or subclinical and thus only 


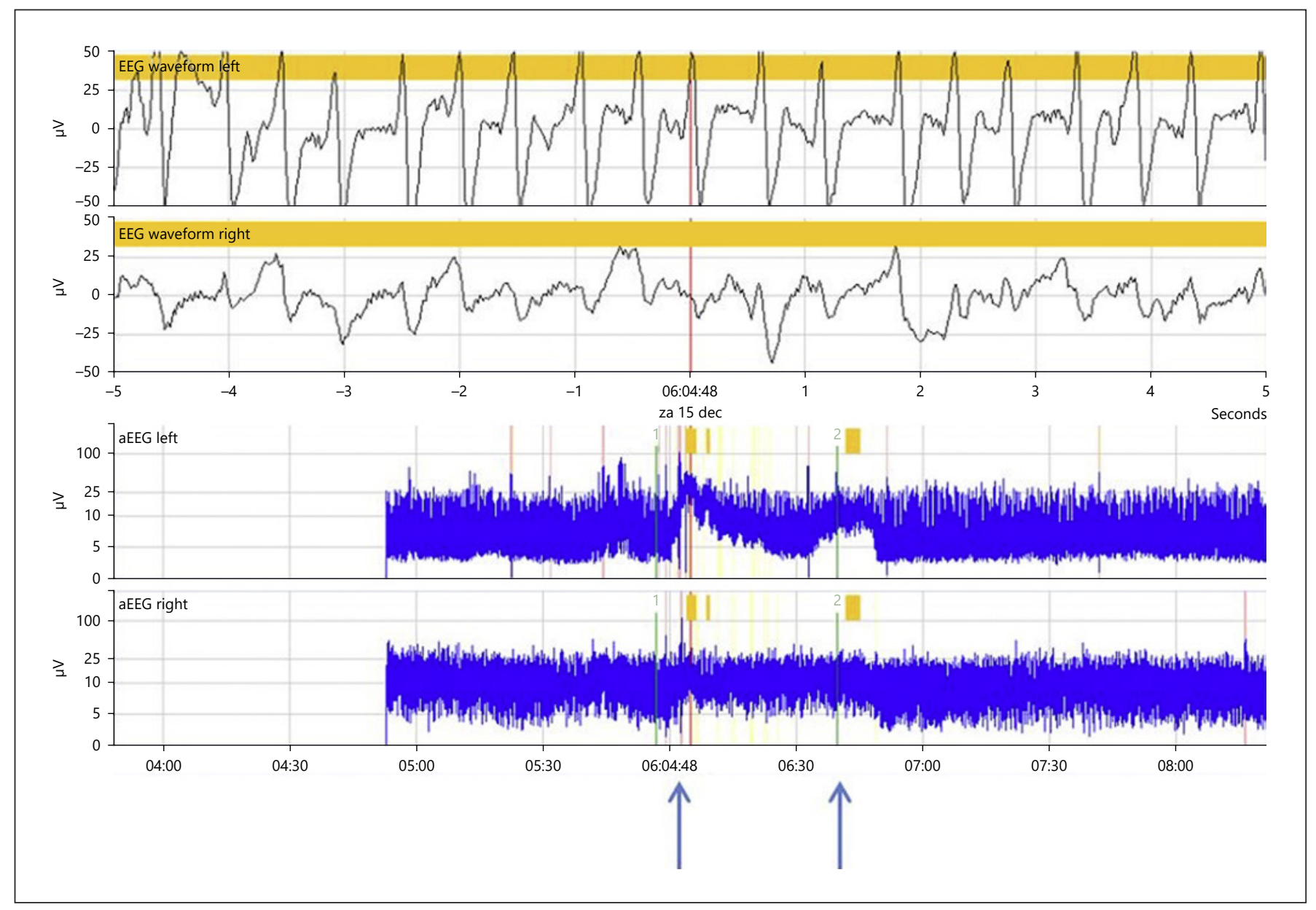

Fig. 1. Example of electrographic seizure on 2-channel aEEG. This figure shows an aEEG trace with an abrupt rise of the lower border (left arrow) on the left side of the brain. The corresponding raw EEG trace shows a rhythmic evolving pattern. The yellow marking indicates that the seizure is detected by the SDA. The right arrow, pointing towards the line marked by the number 2, is the time of AED treatment.

visible when electrographically recorded [4]. Use of electroencephalography (EEG) or amplitude-integrated EEG (aEEG) is required for accurate diagnosis $[5,6]$. As there is evidence for seizures causing further brain damage, it is generally accepted that seizures and the underlying cause should be treated as soon as possible. The longer seizures last, the harder they are to control $[4,7,8]$. However, little is known about current practice with regard to the time of administration of anti-epileptic drugs (AEDs) [9]. In this study, we reviewed the timing of AED treatment in a level III neonatal intensive care unit (NICU) with long-standing expertise in using aEEG and a policy of active seizure treatment. Second, factors associated with recognition of seizures and delay of treatment by the clinician were determined.

\section{Methods}

Setting and Population

Full-term infants (gestational age $\geq 37$ weeks) with aEEG-confirmed seizures, treated with AEDs at the level III NICU of the Wilhelmina Children's Hospital (2012-2017), were studied retrospectively. Infants with neonatal seizures were selected using a local database on discharge diagnosis.

Outcome Measures

The primary outcome of this study was the time interval between the first aEEG-confirmed seizure by the researcher and the initiation of AED treatment. For confirming electrographic seizures, 2-channel aEEG with corresponding raw EEG was used. Secondary, the group treated within $1 \mathrm{~h}$ and the group treated after $1 \mathrm{~h}$ were investigated for differences in the following: seizure characteristics (clinical signs, frequency, duration, and whether the seizure was easy to recognise on the aEEG trace), occurrence during 
Table 1. Patient characteristics

\begin{tabular}{lc}
\hline & Cohort $(n=106)$ \\
\hline Sex, male/female, $n$ & $63 / 43$ \\
Gestational age, weeks+days, mean (range) & $39+6(37+0-42+4)$ \\
Birth weight, g, mean (range) & $3,478(1,900-4,875)$ \\
Apgar at 1 min, median (IQR) & $6(2-9)$ \\
Apgar at 5 min, median (IQR) & $8(4-10)$ \\
Neonatal deaths, $n$ (\%) & $30(28.3)$ \\
Diagnosis, $n(\%)$ & \\
HIE & $46(43.3)$ \\
Stroke (PAIS, CSVT) & $17(16.0)$ \\
ICH & $15(14.2)$ \\
CNS infection & $11(10.4)$ \\
Metabolic disorder & $6(5.7)$ \\
Genetic disorder (other than metabolic) & $7(6.6)$ \\
Neonatal epilepsy (unknown cause) & $4(3.8)$ \\
Type of AED used, $n$ (\%) & \\
Phenobarbital & $89(84.0)$ \\
Lidocaine & $11(10.4)$ \\
Midazolam & $6(5.7)$
\end{tabular}

HIE, hypoxic ischaemic encephalopathy; PAIS, perinatal arterial ischaemic stroke; CSVT, cerebral sinovenous thrombosis; $\mathrm{ICH}$, intracranial haemorrhage; CNS, central nervous system; AED, anti-epileptic drug.

out-of-office hours, underlying diagnosis, and detection rate by the seizure detection algorithm (SDA) of the BrainZ BRM3 monitor. As prompt treatment is considered beneficial, we used a 1-hour time interval as appropriate treatment, in accordance with previous research [9]. We assessed whether the first aEEG-confirmed seizure reported by the clinician in the patient files was in accordance with the first aEEG-confirmed seizure by the researcher. Factors associated with the time interval between the first aEEGconfirmed seizure recorded in the patient files and the time of AED treatment were assessed.

\section{Data Collection}

Electrographic Monitoring

The aEEG recordings obtained during admission were reviewed by the researchers for seizures and background pattern. The initial review of the aEEGs was performed by the first author. For the majority of the infants the recordings were scored together with an experienced aEEG/EEG reader (L.S.V., L.C.W.). Seizure detection by the SDA, seizure duration in seconds, whether the seizure occurred during office hours, and seizure frequency (single, repetitive, or status epilepticus) were recorded. Twochannel aEEG recordings with raw EEG traces were obtained using the BrainZ monitor, Olympic BrainZ monitor, NicoletOne monitor (all Natus, Seattle, WA, USA) and Nihon Kohden monitor (Nihon Kohden Corp., Tokyo, Japan). The location of the EEG electrodes was F3-P3, F4-P4 according to the 10-20 system. Continuous aEEG recordings are standard care at our NICU for neonates at risk for seizures or with clinically suspected seizures. Medical and nursing staff are well trained and experienced in interpreting the aEEG recordings. Seizures on the aEEG trace were
Table 2. Time interval to initiation of AED treatment

\begin{tabular}{llc}
\hline & Frequencies, $n(\%)$ & Cumulative, \% \\
\hline$<1 \mathrm{~h}$ & $34(32.1)$ & 32.1 \\
$1-2 \mathrm{~h}$ & $21(19.8)$ & 51.9 \\
$2-4 \mathrm{~h}$ & $23(21.7)$ & 73.6 \\
$4-8 \mathrm{~h}$ & $14(13.2)$ & 86.8 \\
$>8 \mathrm{~h}$ & $14(13.2)$ & 100.0 \\
\hline
\end{tabular}

defined as an abrupt and transient rise of the lower border with the corresponding raw EEG showing evolving rhythmic activity with a clear start and end and with a duration of at least $10 \mathrm{~s}$ (Fig. 1).

Background pattern of the aEEG trace was scored as described by Hellström-Westas et al. [10, 11]. Seizures on aEEG were considered easy to recognise when they had a duration longer than 20 $s$, with a clear rise of the lower and upper border of the background pattern, or when they were detected by the SDA, without artefacts on the trace. The timing of treatment was marked by the nurse on the aEEG recordings.

\section{Patient Files}

Patient files were reviewed to see whether the first seizure recognised by the researcher was recorded by the clinician. Information from the patient files was used to determine the underlying diagnosis, type of AED, and whether AED treatment was received before commencement of aEEG recording.

\section{Statistical Analysis}

Acquired data were analysed using SPSS version 25 (IBM Corp., Armonk, NY, USA). The difference between the group treated within $1 \mathrm{~h}$ and the group treated after $1 \mathrm{~h}$ was compared with Pearson chi-squared or Fisher's exact test for categorical data and Mann-Whitney U test for continuous data. Multivariable logistic regression analysis was used to assess which factors were associated with the first aEEG seizure recorded by the clinician being equal to the first aEEG seizure confirmed by the researcher.

\section{Results}

During the study period, 165 full-term infants had a discharge diagnosis including seizures. Of these infants, 57 had no seizures on aEEG, but for 16 of them, AED treatment for clinical seizures in the referral hospital was documented and another 6 of them received AEDs even though there was no seizure activity confirmed when reviewing the recording. In 2 cases, infants had seizures on aEEG confirmed by the researcher but were not treated with AEDs.

In this study, 106 full-term infants were included. Patient characteristics are shown in Table 1. Of the aEEGconfirmed first seizures, 34/106 (32.1\%) were treated 
Table 3. Seizure characteristics and differences between seizures treated within and after $1 \mathrm{~h}$

\begin{tabular}{lcccc}
\hline & $\begin{array}{l}\text { Cohort } \\
(n=106)\end{array}$ & $\begin{array}{l}<1 \mathrm{~h} \\
(n=34)\end{array}$ & $\begin{array}{l}>1 \mathrm{~h} \\
(n=72)\end{array}$ & $p$ value \\
\hline Detection by SDA*, $n(\%)$ & $48(50.5)$ & $22(66.7)$ & $26(41.9)$ & 0.22 \\
Clinical signs, $n(\%)$ & $54(50.9)$ & $27(79.4)$ & $27(37.5)$ & $<0.001$ \\
Seizures during office hours, $n(\%)$ & $24(22.6)$ & $8(23.5)$ & $16(22.2)$ & 0.88 \\
Easy to recognize on aEEG trace, $n(\%)$ & $87(82.1)$ & $32(94.1)$ & $55(76.4)$ & 0.03 \\
Seizure duration, s, median (IQR) & $89(43-224)$ & $137(58-546)$ & $70(39-173)$ & 0.02 \\
Seizure frequency, $n(\%)$ & & & & 0.10 \\
$\quad$ Single & $16(15.1)$ & $8(23.5)$ & $8(11.1)$ & 0.55 \\
$\quad$ Repetitive & $82(77.4)$ & $22(64.7)$ & $60(83.3)$ & $82(77.4)$ \\
$\quad$ Status epilepticus & $8(7.5)$ & $4(11.8)$ & $33(55.0)$ & \\
Background pattern**, $n(\%)$ & $43(40.6)$ & $10(43.5)$ & $6(10.0)$ & \\
CNV & $21(19.8)$ & $8(34.8)$ & $3(13.3)$ \\
DNV & $9(8.5)$ & $3(13.0)$ & $2(8.7)$ & \\
BS & $10(9.4)$ & & & \\
FT & & & \\
\hline
\end{tabular}

* 11 cases did not have SDA. ${ }^{* *}$ For 23 cases, the background pattern could not be determined because of presence of status epilepticus, repetitive seizures, or artefacts that did not allow for scoring of the background pattern. SDA, seizure detection algorithm; aEEG, amplitude-integrated electroencephalography; IQR, interquartile range; $\mathrm{CNV}$, continuous normal voltage; DNV, discontinuous normal voltage; BS, burst suppression; FT, flat trace.

within $1 \mathrm{~h}$. Frequencies of treatment within different time intervals are shown in Table 2.

The median time interval was $1: 50 \mathrm{~h}$ with an interquartile range (IQR) from 0:43 to 4:30 h. When we compared this to the time interval between the first aEEG seizure recorded by the clinician and the time of AED treatment, the time intervals are shorter, with 40/106 (37.7\%) seizures treated within $1 \mathrm{~h}$ and only $2 / 106(1.9 \%)$ treated after $8 \mathrm{~h}$, with a median of 1:25 $\mathrm{h}$ and an IQR from 0:40 to $2: 23 \mathrm{~h}$. Outliers with long time intervals occurred for cases in which the first electrographic seizure was not recognised or acted on by the clinician. In 48/106 (45.3\%) cases an AED had already been administered in a level II hospital, prior to transfer to the level III centre and before commencement of aEEG recording.

Baseline characteristics for the group treated within 1 $\mathrm{h}$ and the group treated after $1 \mathrm{~h}$ showed no significant differences. When SDA was available, detection rate was higher for seizures treated within $1 \mathrm{~h}$ compared to those treated after $1 \mathrm{~h}(66.7$ vs. $41.9 \%, p=0.02)$. The seizures that were treated within $1 \mathrm{~h}$ were more often associated with clinical symptoms (79.4 vs. $37.5 \%, p<0.001)$, were easy to recognize on the aEEG trace ( 94.1 vs. $76.4 \%, p=$ 0.03 ), and had a longer median seizure duration (median 137 vs. $70 \mathrm{~s}, p=0.02$; Table 3 ).

In $70.8 \%$, the first seizure confirmed on the aEEG by the researchers corresponded with the first seizure re- corded by the clinician. In the univariate logistic regression analysis, clinical signs and seizures scored easy to recognise on the aEEG were associated with recognition of the first electrographic seizure by the clinician. In the multivariable logistic regression model, clinical signs together with detection by the SDA showed significant association with the first aEEG seizure being recognised by the clinician (Table 4). In 8 cases, the first seizure was recognised by the clinician, but a decision was made to wait for a second aEEG-confirmed seizure to start AED.

\section{Discussion}

The results of this retrospective study show that $32.1 \%$ of the electrographically confirmed first seizures in neonates, admitted to our level III NICU, were treated within a 1-hour time frame, with a median time interval for the whole cohort of 1:50 h. Seizures treated within $1 \mathrm{~h}$ were more often associated with clinical signs, easier to recognise on the aEEG trace, of longer duration, and more often recognised by the SDA compared to seizures treated after $1 \mathrm{~h}$. The time interval to treatment was not significantly different for seizures occurring during out-of-office hours, which reflects the expertise of the medical and nursing staff in interpretation of the aEEG, partly as a result of an aEEG e-learning in the NICU. The first sei- 
Table 4. Factors associated with recording of the first seizure by clinician

\begin{tabular}{lcl}
\hline Cohort $(n=106)$ & Odds ratio & $95 \%$ CI \\
\hline Univariate & & \\
Detection by SDA $\left(n=95^{*}\right)$ & 2.4 & $0.9-5.9$ \\
Clinical signs & 9.8 & $3.4-28.5$ \\
First seizure during out-of-office hours & 1.0 & $0.4-2.7$ \\
Easy to recognize on aEEG trace & 4.6 & $1.6-13.0$ \\
Seizure frequency & & \\
$\quad$ Single & Reference & \\
$\quad$ Repetitive & 0.7 & $0.2-2.4$ \\
$\quad$ Status epilepticus & 2.3 & $0.2-25.2$ \\
Background pattern $\left(n=83^{* *}\right)$ & & \\
$\quad$ CNV & Reference & \\
$\quad$ DNV & 2.5 & $0.8-8.2$ \\
$\quad$ BS & 1.6 & $0.3-7.2$ \\
$\quad$ FT & 1.8 & $0.4-8.1$ \\
Seizure duration & 1.0 & $0.7-1.4$ \\
First AED administered at level III & & \\
$\quad$ centre $\left(n=105^{* * *}\right)$ & 1.4 & $0.6-3.2$ \\
\hline Multivariable $\left(n=95^{*}\right)$ & & \\
Detection by SDA & 4.1 & $1.3-12.4$ \\
Clinical signs & 16.8 & $4.7-59.7$ \\
\hline
\end{tabular}

* 11 cases missing due to unavailability of the SDA. ** 23 cases missing due to unclear background pattern. ${ }^{* * *} 1$ case missing due to unavailability of information on former AED administration. CI, confidence interval; SDA, seizure detection algorithm; aEEG, amplitude-integrated electroencephalography; CNV, continuous normal voltage; DNV, discontinuous normal voltage; BS, burst suppression; FT, flat trace; AED, anti-epileptic drug.

zure that was confirmed by the researcher corresponded with the first seizure recorded in the patient files in $70.8 \%$ of the cases. Main factors associated with recognition of the first seizure by the clinician were detection by the SDA alongside clinical signs.

Literature on timing of AED treatment is scarce. Previously reported treatment of electrographic seizures within $1 \mathrm{~h}$ in the ANSeR study was $11 \%$ [9]. For this study, continuous multi-channel EEG (cEEG) was used instead of aEEG. Other studies using continuous multi-channel EEG, such as those by Low et al. [12] and Lynch et al. [13, 14], showed a wide range in the time interval between electrographically confirmed seizures and AED treatment for small samples of neonates with seizures due to hypoxic ischaemic encephalopathy. The results found in our study show a higher percentage of the electrographically confirmed seizures being treated within $1 \mathrm{~h}$. Even though there is little evidence yet which shows the positive effect of anti-epileptic medication on subsequent outcome in neonatal seizures, there is increasing support to suggest that earlier intervention with subsequent reduced seizure burden reduces MRI injury and is associated with a better outcome [15-18]. Bearing in mind that rapidly controlling seizures makes them easier to treat and could protect the brain from further damage, there is definitely still room for improvement with regard to time to treatment.

Of the first seizures, $50.5 \%$ were detected by the SDA and $50.9 \%$ were associated with clinical signs. A significantly higher percentage of detection for seizures treated within $1 \mathrm{~h}$ demonstrates how using an SDA improves the time to treatment. Furthermore, seizure detection together with clinical signs account for better recognition of seizures by the clinician. This confirms the value of SDA systems as demonstrated in earlier studies and calls for further development of these detection algorithms, increasing detection rates and decreasing false positive detection [19].

Previous studies showed the discrepancy between clinical symptoms taken for seizure activity and the actual electrographic activity recorded by cEEG or aEEG, especially when uncoupling of clinical and electrographic seizures occurs after AED treatment [5, 20, 21]. Therefore, depending on clinical seizures alone is unreliable and awareness of subclinical seizures is essential. Several studies have shown that treatment of clinical and subclinical seizures in neonates with hypoxic ischaemic encephalopathy reduces seizure burden, as well as an association between higher seizure burden and additional brain injury on magnetic resonance imaging, underlining the importance of treatment of electrographic seizures $[15,18]$.

Easy recognition of seizures on the aEEG trace and longer duration of seizures were related to treatment within a 1-hour time interval as well. Indeed, seizures with a longer duration, therefore likely better visible on aEEG, are recognised sooner by the staff as well as the SDA and consequently acted on faster [19]. Previous research showed that seizures of short duration and with low amplitude on the aEEG are more often missed and repetitive seizures are more often recognised [22, 23]. In this study, seizures with shorter duration were not less often recorded in the files, but only acted on later by the clinician. This may have partly been due to a policy to wait for a second aEEG-confirmed seizure before initiating treatment.

This study used 2-channel aEEG with raw EEG for continuous electrographic monitoring of brain activity. Even though cEEG is the gold standard for diagnosing seizures, it requires interpretation by specialists, which is 
not available 24/7 in most centres. Therefore, in a clinical setting, seizures on cEEG are sometimes left untreated as well, or uncertainty about electrographic seizures leads to overtreatment [9]. The use of aEEG combined with raw EEG has increased the accuracy of seizure treatment, with electrographic confirmation of clinical seizures and more subclinical seizures being treated than when treatment is based on clinical signs alone [24]. The use of aEEG in this study shows a higher seizure treatment rate within $1 \mathrm{~h}$ than the ANSeR study, where cEEG was used for monitoring [9]. This attests for the use of aEEG as an addition to multi-channel EEG for diagnosing seizures, to reduce delay in treatment. We do, however, realize that this applies to the seizures that we were able to recognise with aEEG. Furthermore, part of the delay in the ANSeR study could be explained by the variability of expertise in this multi-centre study. Training of medical and nursing staff on accurate interpretation of the aEEG is key to accuracy of seizure detection and subsequent timely treatment.

The main limitation of this study is its retrospective nature. Short single seizures are not in all cases treated, sometimes a "wait and see" policy for a consecutive seizure was implemented. Prospective studies in this setting could clarify the course of action of the clinician. However, it would be challenging to get unbiased results due to raised attention of the clinician to pursue early treatment. Another point to consider here is the varying sensitivity and specificity of limited-channel aEEG as a tool for diagnosing seizures, as a result of which brief lowamplitude electrographic seizures may have been missed $[25,26]$.

In this study, the time between the first aEEG-confirmed seizure and initiated AED treatment was evaluated in a relatively large cohort, making it a reliable representation of timing of AED treatment in our level III centre. Time intervals might be different in centres that use cEEG, no continuous monitoring, or have less experience with the interpretation of aEEG. Inter-observer differences have been reported, depending on the expertise of the aEEG reader $[27,28]$. For this reason, it is possible that the delay in starting treatment will be even longer in centres with less expertise. Yet this study shows that the use of aEEG with SDA by trained personnel could shorten time intervals to treatment compared to other methods and is therefore relevant to centres with availability of 2-channel aEEG.

In this study, we address the timing of AED treatment and factors influencing seizure recognition and delay of treatment. Future research should ascertain whether early treatment is beneficial for short-term (seizure burden) and long-term outcomes (morbidity, disability). The effect of early treatment on prognosis can help clinicians to specify an appropriate time frame for seizure treatment.

\section{Conclusion}

In our population only $32.1 \%$ of the first seizures confirmed on aEEG were treated within $1 \mathrm{~h}$. Even though this is a higher treatment rate within $1 \mathrm{~h}$ than has been reported previously, there is room for improvement. Clinical signs and detection by the SDA play a major role in prompt treatment of seizures. The development of SDAs with a higher accuracy and training staff vigorously to recognise subclinical seizures can help improve recognition and treatment. Monitoring with aEEG is useful for treatment of seizures $24 / 7$, especially at sites where cEEG cannot be assessed continuously, resulting in delayed diagnosis and treatment. Future research is needed to point out how fast treatment needs to be initiated to accomplish optimal outcomes.

\section{Acknowledgements}

The authors thank Joke Zoet-Lavooi, who developed an elearning for aEEG, and all nurses of the NICU of the Wilhelmina Children's Hospital for their dedication in performing aEEG recordings and the precise annotation of events, including the start of anticonvulsants.

\section{Statement of Ethics}

A waiver of informed consent was obtained from the hospital's medical Ethics Committee for this retrospective data analysis. We confirm that we have read the Journal's position issues involved in ethical publication and affirm that this report is consistent with those guidelines.

\section{Conflict of Interest Statement}

Two EEG machines were provided to the department as part of a clinical trial, the NicoletOne for the NEMO project (European Community's 7th Framework Program, HEALTH-F5-2009-4.2-1, grant agreement No. 241479, the NEMO project) and the Nihon Kohden monitor for the ANSeR study funded by a Welcome Trust Strategic Translational Award (098983).

\section{Funding Sources}

No funding was received for this retrospective cohort study. 


\section{Author Contributions}

The research design was done by W.M.J.A., L.S.V., and L.C.W. The aEEGs were reviewed by W.M.J.A., L.S.V., M.C.T., and L.C.W. Acquisition of other data was performed by W.M.J.A. The authors
W.M.J.A., L.C.W., and F.G. were responsible for statistical analysis and interpretation. The article was written by W.M.J.A. and critically revised and approved for publication by L.S.V., M.C.T., F.G., and L.C.W.

\section{References}

1 Volpe JJ. Neonatal seizures. In: Neurology of the newborn. 5th edition. Philidelphia: W.B. Saunders; 2008. pp. 203-44.

2 Tekgul H, Gauvreau K, Soul J, Murphy L, Robertson R, Stewart J, et al. The current etiologic profile and neurodevelopmental outcome of seizures in term newborn infants. Pediatrics. 2006 Apr;117(4):1270-80.

3 Weeke LC, Groenendaal F, Toet MC, Benders MJ, Nievelstein RA, van Rooij LG, et al. The aetiology of neonatal seizures and the diagnostic contribution of neonatal cerebral magnetic resonance imaging. Dev Med Child Neurol. 2015 Mar;57(3):248-56.

4 Glass HC, Wirrell E. Controversies in neonatal seizure management. J Child Neurol. 2009 May;24(5):591-9.

5 Murray DM, Boylan GB, Ali I, Ryan CA, Murphy BP, Connolly S. Defining the gap between electrographic seizure burden, clinical expression and staff recognition of neonatal seizures. Arch Dis Child Fetal Neonatal Ed. 2008 May;93(3):F187-91.

6 Levene M. The clinical conundrum of neonatal seizures. Arch Dis Child Fetal Neonatal Ed. 2002 Mar;86(2):F75-7.

7 Toet MC, Groenendaal F, Osredkar D, van Huffelen AC, de Vries LS. Postneonatal epilepsy following amplitude-integrated EEGdetected neonatal seizures. Pediatr Neurol. 2005 Apr;32(4):241-7.

8 Ronen GM, Buckley D, Penney S, Streiner DL. Long-term prognosis in children with neonatal seizures: a population-based study. Neurology. 2007 Nov;69(19):1816-22.

9 Rennie JM, de Vries LS, Blennow M, Foran A, Shah DK, Livingstone V, et al. Characterisation of neonatal seizures and their treatment using continuous EEG monitoring: a multicentre experience. Arch Dis Child Fetal Neonatal Ed. 2019 Sep;104(5):F493-501.

10 Hellström-Westas L, Rosén I, de Vries L, Griesen G. Amplitude-integrated EEG classification and interpretation in preterm and term infants. Neoreviews. 2006;7(2):e76-87.
11 Abend NS, Wusthoff CJ. Neonatal seizures and status epilepticus. J Clin Neurophysiol. 2012 Oct;29(5):441-8.

12 Low E, Boylan GB, Mathieson SR, Murray DM, Korotchikova I, Stevenson NJ, et al. Cooling and seizure burden in term neonates: an observational study. Arch Dis Child Fetal Neonatal Ed. 2012 Jul;97(4):F267-72.

13 Lynch NE, Stevenson NJ, Livingstone V, Murphy BP, Rennie JM, Boylan GB. The temporal evolution of electrographic seizure burden in neonatal hypoxic ischemic encephalopathy. Epilepsia. 2012 Mar;53(3):549-57.

14 Lynch NE, Stevenson NJ, Livingstone V, Mathieson S, Murphy BP, Rennie JM, et al. The temporal characteristics of seizures in neonatal hypoxic ischemic encephalopathy treated with hypothermia. Seizure. 2015 Dec;33:605.

15 Shah DK, Wusthoff CJ, Clarke P, Wyatt JS, Ramaiah SM, Dias RJ, et al. Electrographic seizures are associated with brain injury in newborns undergoing therapeutic hypothermia. Arch Dis Child Fetal Neonatal Ed. 2014 May;99(3):F219-24.

16 Srinivasakumar P, Zempel J, Trivedi S, Wallendorf M, Rao R, Smith B, et al. Treating EEG Seizures in Hypoxic Ischemic Encephalopathy: A Randomized Controlled Trial. Pediatrics. 2015 Nov;136(5):e1302-9.

17 Kharoshankaya L, Stevenson NJ, Livingstone V, Murray DM, Murphy BP, Ahearne CE, et al. Seizure burden and neurodevelopmental outcome in neonates with hypoxic-ischemic encephalopathy. Dev Med Child Neurol. 2016 Dec;58(12):1242-8.

18 van Rooij LG, Toet MC, van Huffelen AC, Groenendaal F, Laan W, Zecic A, et al. Effect of treatment of subclinical neonatal seizures detected with aEEG: randomized, controlled trial. Pediatrics. 2010 Feb;125(2):e358-66.

19 Mathieson S, Rennie J, Livingstone V, Temko A, Low E, Pressler RM, et al. In-depth performance analysis of an EEG based neonatal seizure detection algorithm. Clin Neurophysiol. 2016 May;127(5):2246-56.
20 Biagioni E, Ferrari F, Boldrini A, Roversi MF, Cioni G. Electroclinical correlation in neonatal seizures. Eur J Paediatr Neurol. 1998;2(3): 117-25.

21 Scher MS, Alvin J, Gaus L, Minnigh B, Painter MJ. Uncoupling of EEG-clinical neonatal seizures after antiepileptic drug use. Pediatr Neurol. 2003 Apr;28(4):277-80.

22 Toet MC, van der Meij W, de Vries LS, Uiterwaal CS, van Huffelen KC. Comparison between simultaneously recorded amplitude integrated electroencephalogram (cerebral function monitor) and standard electroencephalogram in neonates. Pediatrics. 2002 May;109(5):772-9.

23 Shellhaas RA, Soaita AI, Clancy RR. Sensitivity of amplitude-integrated electroencephalography for neonatal seizure detection. Pediatrics. 2007 Oct;120(4):770-7.

24 Shellhaas RA, Barks AK. Impact of amplitude-integrated electroencephalograms on clinical care for neonates with seizures. Pediatr Neurol. 2012 Jan;46(1):32-5.

25 Rakshasbhuvankar A, Paul S, Nagarajan L, Ghosh S, Rao S. Amplitude-integrated EEG for detection of neonatal seizures: a systematic review. Seizure. 2015 Dec;33:90-8.

26 Shah DK, Mackay MT, Lavery S, Watson S, Harvey AS, Zempel J, et al. Accuracy of bedside electroencephalographic monitoring in comparison with simultaneous continuous conventional electroencephalography for seizure detection in term infants. Pediatrics. 2008 Jun;121(6):1146-54.

27 Mehta B, Griffiths N, Spence K, Laing S. Interobserver reliability in reading amplitude-integrated electroencephalogram in the newborn intensive care unit. J Paediatr Child Health. 2017 Oct;53(10):1007-12.

28 Rennie JM, Chorley G, Boylan GB, Pressler R, Nguyen Y, Hooper R. Non-expert use of the cerebral function monitor for neonatal seizure detection. Arch Dis Child Fetal Neonatal Ed. 2004 Jan;89(1):F37-40. 